\title{
Assessment of dermatologists' perception of utilizing teledermatology during COVID-19 pandemic in Saudi Arabia
}

Hatoun M. Almaziad, MD, Abdulrahman I. Alfawzan, MD, Norah K. Alkhayal, MD, Rayan A. Alkhodair, MD, FRCPC.

\begin{abstract}
الأهداف : تقييم تصور أطباء الأمراض الجلدية في المملكة العربية السعودية

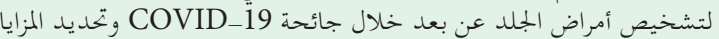

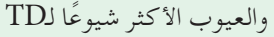

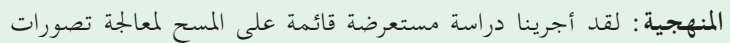

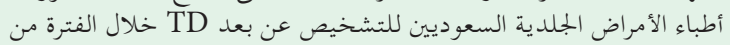

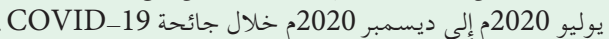

النتائج: من بين 664 رسالة بريد إِكتروني مرسله، استجاب 107 بإِجابة

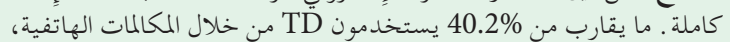

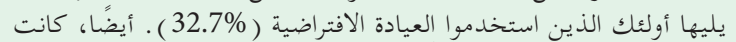

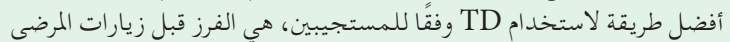

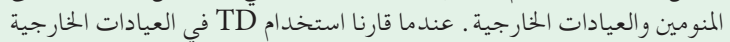

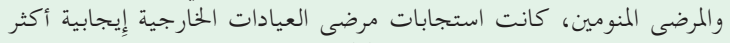

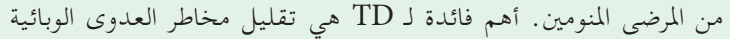

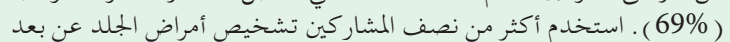

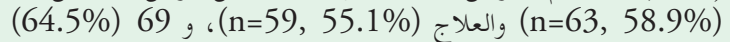
اعتبروا استخدامه في المستقبل.

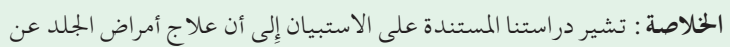

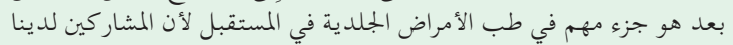

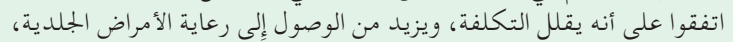

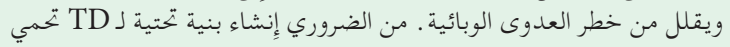

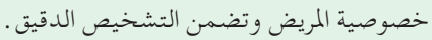

Objectives: To assess teledermatology (TD) perception among dermatologists in the Kingdom of Saudi Arabia during the COVID-19 pandemic and to identify the most common advantages and disadvantages of TD.

Methods: We conducted a cross-sectional surveybased study to address Saudi dermatologist perceptions of TD from July 2020 to December 2020, during Covid-19 pandemic.

Results: Out of 664 emailssent, 107 complete responses were returned. Approximately $40.2 \%$ usedTD through phone calls, followed by those who used a virtual clinic $(32.7 \%)$. Also, the best way to use TD, according to respondents, was for triage before inpatient and outpatient visits. When we compared the use of TD in the outpatient and inpatient settings, outpatient responses always had more positive attitudes than inpatients. The most important benefit of TD is to reduce the risk of pandemic infections (69\%). More than half of the participants sometimes used TD for diagnosis $(n=63,58.9 \%)$ and management $(n=59$, $55.1 \%)$, and $69(64.5 \%)$ considered using it in the future.

Conclusion: Our survey-based study indicates that $\mathrm{TD}$ is an important part in the future dermatology because our participants agreed that TD decreases cost, increases access to dermatology care, and reduces the risk of pandemic infections. And it is necessary to establish an infrastructure for TD that protects patient's privacy and ensures accurate diagnosis.

Keywords: Teledermatology, Saudi Arabia, COVID-19

Saudi Med J 2021; Vol. 42 (9): 1024-1030 doi: 10.15537/smj.2021.42.9.20210342

From the Department of Dermatology and Dermatological Surgery (Almaziad), Prince Sultan Military Medical City; from the College of Medicine (Alfawzan, Alkhodair), King Saud University for Health Sciences; and from the Department of Medicine (Alkhayal, Alkhodair), Division of Dermatology, King Abdulaziz Medical City, Riyadh, Kingdom of Saudi Arabia.

Received 11th May 2021. Accepted 22nd July 2021.

Address correspondence and reprint request to: Dr. Abdulrahman I. Alfawzan, College of Medicine, KingSaud University for Health Sciences, Riyadh, Kingdom of Saudi Arabia. E-mail: alfawzan.job@gmail.com ORCID ID: https://orcid.org/0000-0003-1508-3710 
$\mathrm{R}^{\mathrm{e}}$ ecent advances in technology have opened enormous access to different services across the medical field. One of the most important implementations of technology in recent years is telemedicine. Teledermatology (TD) is defined as patient consultation, which overcomes the challenge of location and time. Teledermatology could be conducted as both a store and forward (SF) and a live consultation. ${ }^{1}$ Store and forward involves uploading and saving patient data, such as photographs and laboratory results, with a brief history for later consultation by a dermatologist, while live consultations consist of a synchronous connection with a dermatologist, allowing for real-time responses. Teledermatology has been widely used in patients' triage, providing services to patients in rural areas, and for follow-up care. One of the most important advantages of TD is alleviating the economic burden for the health care system and patients. ${ }^{2,3}$ For example, reducing patient travel time results in fewer absent days from work due to hospital visits, which is particularly important for dermatology patients since most of the appointments are for follow-up care. ${ }^{3}$ Teledermatology has also been used as a beneficial educational tool for residents and students. ${ }^{4}$

In March 2020, the World Health Organization (WHO) declared COVID-19 as a worldwide pandemic. ${ }^{5}$ The social distancing protocols created in response to the pandemic have faced dermatologists with the challenge of reducing exposure risk themselves and patients while still providing high-quality care. The American Academy of Dermatology provides a guide to implementing TD during the crisis. ${ }^{6}$ In addition, violations of the Health Insurance Portability and Accountability Act (HIPAA) from practitioners using TD will be forgiven during the crisis. In the Kingdom of Saudi Arabia (KSA), most tertiary hospitals have rapidly implemented TD to minimize provider and patient exposure risk while ensuring that the health care process continues. However, rapid implementation of TD has been challenging, with many pros and cons. One challenge is instructing patients on how to use TD. Also, technology availability and network coverage remains a major obstacle. ${ }^{7}$ Many patients have concerns

Disclosure. Authors have no conflict of interests, and the work was not supported or funded by any drug company. This study was approved by the Institutional Review Board (IRB), King Abdullah International Medical Research Center (IRB number RC20/378/R). about their privacy and confidentiality, especially since there is no infrastructure built for TD. ${ }^{7,8}$

There are many cross-sectional surveys about dermatologists' perceptions regarding TD. In a systematic literature review conducted by Eissing et al, ${ }^{9}$ most of the studies found that TD is reliable and effective, particularly for triage and remote consultations, such as rural areas and nursing homes. Due to the challenges posed by the COVID-19 pandemic, providers have been forced to apply TD on an unprecedented scale, raising questions about potentially broader usage. In KSA, many dermatologists have supported the use of TD during the pandemic, and many are considering using it in the future. ${ }^{10}$ These trends may lead to major changes in the process of learning and practice. Therefore, this survey-based study aims to assess the perception of inpatient and outpatient TD among dermatologists in KSA during the COVID-19 pandemic and to identify the most common advantages and disadvantages of TD.

Methods. We conducted a nationwide cross-sectional survey-based study from July 2020 to December 2020 to address Saudi dermatologists' perceptions of TD during the Covid-19 pandemic.

This study was conducted in KSA and includes all practicing Saudi dermatologists in KSA. We excluded all other physicians and medical students. As of September 2020, there are 664 practicing dermatology consultants, specialists, and residents. After obtaining ethical approval, an official email was sent from the Saudi Commission for Health Sciences to all registered Saudi dermatologists in KSA.

We used an electronic self-administered questionnaire among all practicing Saudi dermatologists in KSA. The questionnaire included gender, level of dermatologist, years of practicing dermatology, practicing region, private or governmental affiliation, and the method of practicing TD. It also includes perceptions about TD consultations among dermatologists in inpatient and outpatient settings, as well as the advantages and disadvantages of TD consultations from the dermatologist viewpoint. Regarding the comparative assessment of outpatient and inpatient TD and perception of $\mathrm{TD}$, the survey included 5 answers, from 5 (strongly agree) to 1 (strongly disagree). Two questions focused on how often dermatologists used TD for diagnosis and management, which included 5 answers from 1 (never) to 5 (always). The questionnaire was written by experts, and the previous version has been used in previous studies. ${ }^{11}$

Statistical analysis. The data was gathered from the questionnaires in google form and entered into excel sheet. The Statistical Package for Social Sciences (SPSS) 
version 21 (IBM Corp., Armonk, NY, USA) was used for data entering and analysis directly. The categorical variables were presented as frequencies and percentages that included gender, level of dermatologist, region, workplace, TD type, and advantages and disadvantages of TD. The 2 questions focused on how often dermatologists used TD for diagnosis and management and the comparative assessment of outpatient and inpatient TD were presented as mean and standard deviation. The $p$-value for the comparative assessment of outpatient and inpatient patients was calculated by paired t-test, while the $p$-value between levels was calculated by analysis of variance (ANOVA) test.

Before data collection, Institutional Review Board (IRB) approval was obtained from King Abdullah International Medical Research Center.

Results. Out of 664 emails sent to Saudi dermatologists, 111 received responses, with a response rate of $16.7 \%$. We excluded 4 responses for incomplete survey. Out of 107 respondents, more than half are male and half are working in governmental hospitals, while one-third of all respondents work in both the governmental and private sectors. Ninety-nine (92.5\%) used TD during the COVID-19 pandemic, most commonly through phone calls $(\mathrm{n}=43,40.2 \%)$. Ninetythree $(87 \%)$ participants usually answer consultations from relatives through non-institutional channels such as social media applications; this number increased after the COVID-19 pandemic to 100 (93\%; Table 1).

Outpatient and inpatient teledermatology. Regarding the comparative assessment of TD in the outpatient and inpatient settings, most responses were toward the neutral or positive scale (strongly agree and agree). Interestingly, in all 6 items in Table 2, outpatients always had a higher mean (toward the positive scale) than inpatients. Participants are more likely to agree that TD helps overall outpatient care more than inpatient care, with a significant difference $(p<0.05)$. However, when we asked if "TD helps overall patient care if dermatology is not available", no significant difference between inpatient and outpatient patients was observed. A significant difference is also appreciated when we asked if "TD increases access to dermatologic care" $(p<0.05)$ and "TD can be used to triage patients from outpatient to inpatient and vice versa" ( $p=0.016$; Table 2).

Our participants believe that the best way to utilize TD is for triage consults before seeing patients in both outpatient $(n=42,39.2 \%)$ and inpatient $(n=41,38.3)$ settings. Also, $60 \%$ believe that TD will decrease healthcare costs for inpatients and $79 \%$ for outpatients.
Table 1 - Participant characteristics.

\begin{tabular}{lc}
\hline Variables & $\mathbf{n}(\%)$ \\
\hline Gender & $63(58.9)$ \\
Male & $44(41.1)$ \\
Female & \\
Level & $45(42.1)$ \\
Consultant & $23(21.5)$ \\
Specialist & $39(36.4)$ \\
Resident & \\
Region & $63(58.9)$ \\
Central & $10(9.3)$ \\
South & $12(11.2)$ \\
West & $13(12.1)$ \\
East & $9(8.4)$ \\
North & \\
Workplace & \\
Governmental & $53(49.5)$ \\
Private & $23(21.5)$ \\
Both & $31(29)$ \\
Teledermatology type & \\
Phone call & \\
Virtual clinic & $43(40.2)$ \\
Social media & $35(32.7)$ \\
None & $21(19.6)$ \\
Years practicing dermatology, Mean $\pm S D$ & $8(7.5)$ \\
& $10.3 \pm 9.7$ \\
\hline
\end{tabular}

Approximately $50 \%$ of the respondents agreed that TD will decrease response time for consults for both inpatient $(n=53,49 \%)$ and outpatient $(n=56,52 \%)$. Fifty-six percent of the respondents were not satisfied with the utilization of TD in the inpatient setting, in contrast to only $36 \%$ for the outpatient setting.

Outpatient and inpatient teledermatology by level of dermatologist. In Table 3, we compared the answers between the inpatient and outpatient settings by the level of dermatologists. Consultants achieved higher score in that "TD helps inpatient care if dermatology is not available" more than non-consultants with a significant difference between groups ( $p=0.048$ ). Also, a significant difference was noted that TD increases medical liability for both inpatients $(p<0.05)$ and outpatients $(p=0.05)$.

Teledermatology in diagnosis and education. Residents slightly agree that TD should be part of residents' education curriculum (mean $=3.8$ ) more than specialists and consultants (mean=3.6). Also, they are more likely to agree that it affects their ability to do a proper physical examination. However, there was no significant difference in the 4 items regarding different level of dermatologists in Figure 1.

Advantages and disadvantages of teledermatology. More than half of our participants agreed that TD can decrease the risk of pandemic infection (69\%). They see that TD can insure continuous care, especially for patients with chronic disorders. Figure 2 represents 
Table 2 - Outpatient versus inpatient teledermatology.

\begin{tabular}{lccc}
\hline Variables & Outpatient & Inpatient & $P$-value \\
\hline TD overall helps patient care & $4.12 \pm 0.8$ & $3.68 \pm 0.95$ & $<0.05$ \\
TD helps overall patient care if dermatology is not available & $4.29 \pm 0.73$ & $4.17 \pm 0.72$ & 0.051 \\
TD increases access to dermatologic care & $4.15 \pm 0.76$ & $3.79 \pm 0.9$ & $<0.05$ \\
TD increases medical liability & $3.86 \pm 0.9$ & $3.76 \pm 0.84$ & 0.14 \\
TD can be used to triage patients from & $3.94 \pm 0.85$ & $3.74 \pm 0.71$ & 0.016 \\
TD would benefit from a standardized triage algorithm or management approach to provide \\
consistency of care
\end{tabular}

Values are presented as Mean $\pm \mathrm{SD}$, TD: teledermatology

Table 3 - Outpatient and inpatient teledermatology by level.

\begin{tabular}{|c|c|c|c|c|}
\hline \multirow[t]{2}{*}{ Items } & \multicolumn{3}{|c|}{ Mean } & \multirow[t]{2}{*}{$P$-value } \\
\hline & Consultant & Specialist & Resident & \\
\hline Outpatient TD overall helps patient care & 4.26 & 4.04 & 4 & 0.27 \\
\hline Inpatient TD overall helps patient care & 3.73 & 3.69 & 3.61 & 0.85 \\
\hline TD helps overall outpatient care if dermatology is not available & 4.51 & 4.09 & 4.29 & 0.28 \\
\hline TD helps overall inpatient care if dermatology is not available & 4.37 & 4 & 4.05 & 0.048 \\
\hline TD increases access to outpatient dermatologic care & 3.31 & 3.95 & 4.10 & 0.16 \\
\hline TD increases access to inpatient dermatologic care & 3.97 & 3.82 & 3.56 & 0.11 \\
\hline Outpatient TD increases liability & 4.06 & 3.52 & 3.84 & 0.05 \\
\hline Inpatient $\mathrm{TD}$ increases liability & 4 & 3.34 & 3.74 & 0.009 \\
\hline Outpatient TD can be used to triage patients from outpatient to inpatient care & 3.97 & 3.95 & 3.89 & 0.91 \\
\hline Inpatient $\mathrm{TD}$ can be used to triage patients from inpatient to outpatient care & 3.75 & 3.87 & 3.66 & 0.56 \\
\hline $\begin{array}{l}\text { Outpatient TD would benefit from a standardized triage algorithm or management approach } \\
\text { to provide consistency of care }\end{array}$ & 4.11 & 3.91 & 3.79 & 0.16 \\
\hline $\begin{array}{l}\text { Inpatient TD would benefit from a standardized triage algorithm or management approach } \\
\text { to provide consistency of care }\end{array}$ & 3.88 & 3.82 & 3.87 & 0.93 \\
\hline
\end{tabular}

TD: teledermatology. The mean ranges from $1=$ strongly disagree to $5=$ strongly agree.

the most common advantages of TD. However, the most common concern regarding $\mathrm{TD}$ is the possible misunderstandings of the transmitted information (Figure 3).

Teledermatology for diagnosis, management, and future practice. More than half of respondents sometimes use TD for diagnosis $(\mathrm{n}=63,58.9 \%)$ and management $(n=59,55.1 \%)$, and there was no significant difference between levels. About two-thirds of dermatologists have considered using TD $(n=69,64.5 \%)$; however, the majority of participants who do not want to use TD are residents $(71 \%)$.

Discussion. Our respondents think that the best way to utilize TD is for triage before seeing patients in both inpatient and outpatient settings. Appropriate triage of patients can decrease a patient's time spent waiting to see a dermatologist. It can decrease travel time to the hospital and will improve patient care. It can also reduce the risk of transmission of COVID-19 to patients and dermatologists. Barbieri et al, ${ }^{12}$ compared 2 independent teledermatologists with one in-person dermatologist triaging. In-person dermatologist triaged $66 \%$ of the patients to the next day or later, while 2 independent teledermatologists each triaged $60 \%$ of the patients to the next day or later. Most of our respondents believe that TD can slightly reduce health care costs in the outpatient setting $(79 \%)$ more than the inpatient setting (60\%). It is beneficial for the outpatient clinic, since implementing TD will decrease patient travel time, waiting time, and absences from work. ${ }^{3}$ In a review of 11 studies, 9 found that TD is more cost effective than face-to-face (FTF) consultations. Also, 6 studies found it is beneficial for patients to be triaged before being seen. ${ }^{13}$

The most common advantage of TD is reducing the risk of pandemic infection. According to Yeroushalmi et $\mathrm{al}^{14} 73.6 \%$ of the patients liked TD because they 


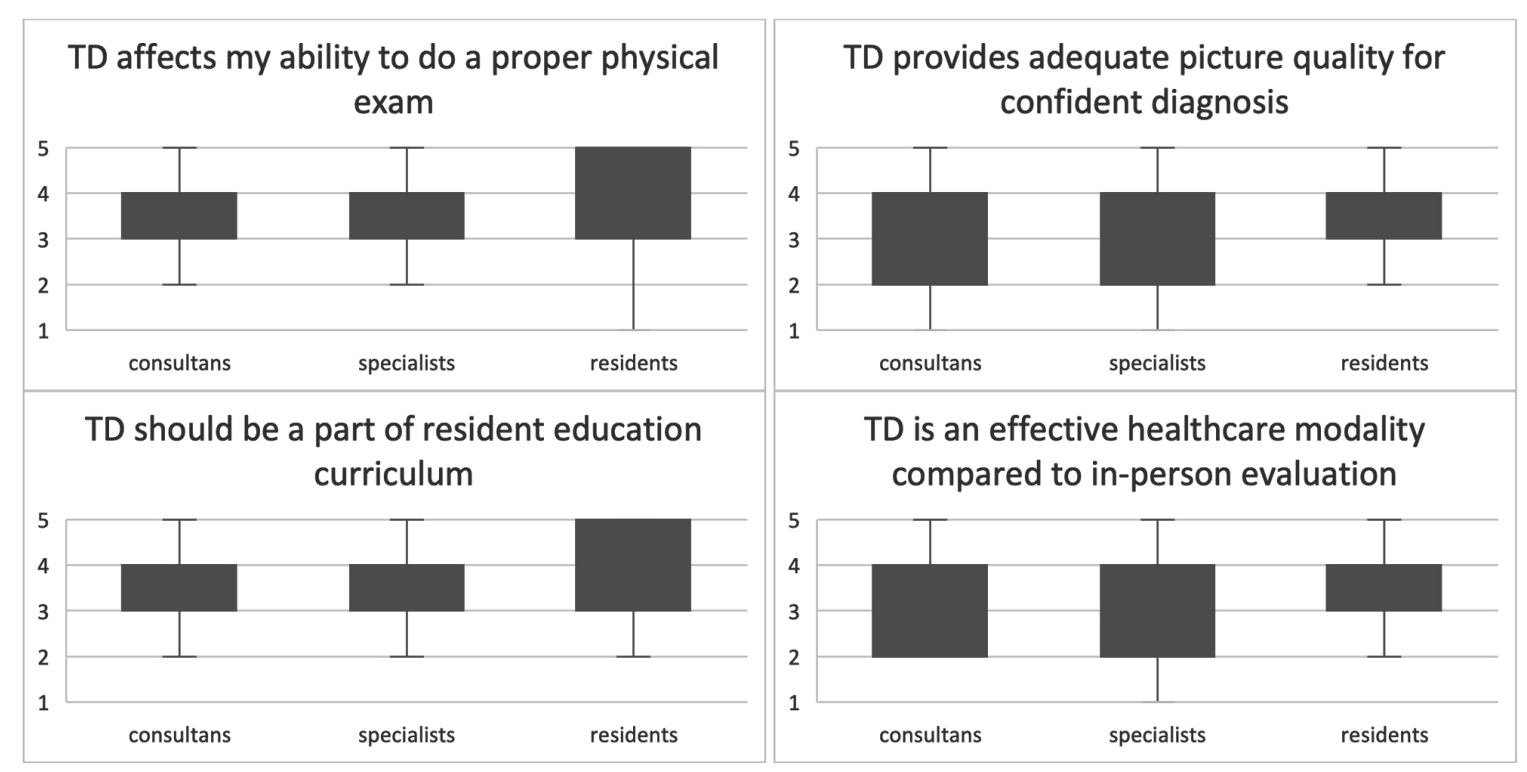

Figure 1 - Dermatologists perception of teledermatology. 5= strongly agree and 1= strongly disagree. TD: teledermatology.

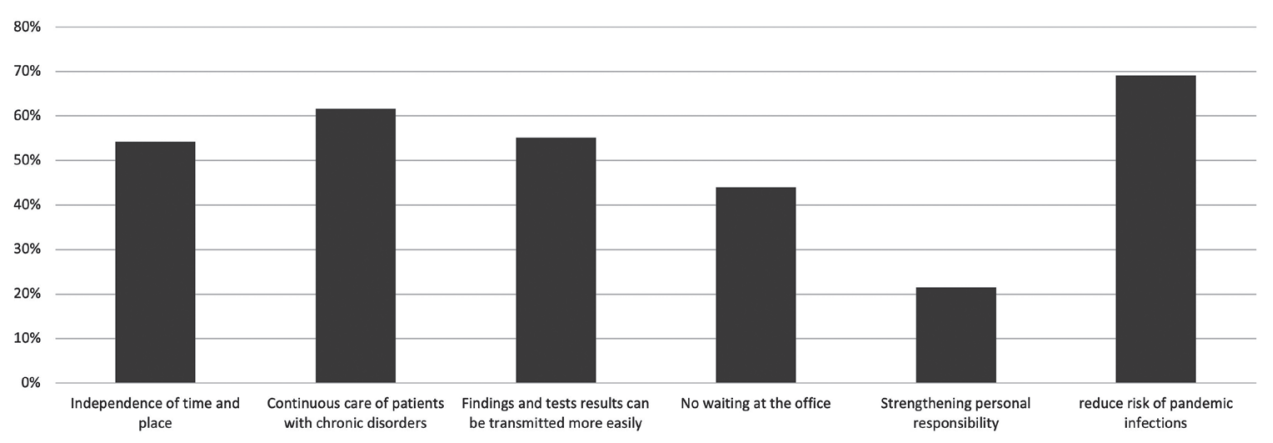

Figure 2 - Advantages of teledermatology.

received the care they needed while maintaining social distancing. Also, another study among dermatologists found that $78 \%$ of the respondents felt that they were participating in efforts to decrease in-person contact during the COVID-19 pandemic. ${ }^{15}$ More than 50\% of our respondents think that results can transmit easily and cite independence of time and place as advantages. Perhaps one of the most important benefits of TD is the expansion of dermatological care to rural areas. In the latest Ministry of Health $(\mathrm{MOH})$ statistical yearbook 2018, the number of dermatologists in Riyadh, KSA, was 9.2 per 100,000 people in comparison to Najran, KSA, which was 3.18. ${ }^{16}$ This significant maldistribution can be overcome by implementing TD.

The most common disadvantage cited among our respondents is the possible misunderstanding of the transmitted information, followed by lack of personal contact. Our results are similar to previous surveybased studies. ${ }^{17,18}$ However, many studies have found that there is a high concordance between in-person and TD consultation. ${ }^{19}$ Gabel et al ${ }^{20}$ found that out of 41 cases, there was a high agreement between FTF and TD consultations concerning diagnosis with differentials $($ Kappa $=0.83)$ and management $(\mathrm{Kappa}=1.0)$ of the cases.

According to Alakeel, ${ }^{10}$ patient refusal to participate in TD was the least common reason for dermatologists to not practice TD. However, a study conducted in KSA assessing patient's satisfaction with TD showed that $14 \%$ of patients refused to provide photographs for religious and social reasons. ${ }^{21}$ This is somewhat in accordance with our study where dermatologists thought that data security is a major disadvantage. Technical issues and lack of internet connection are other main obstacles 


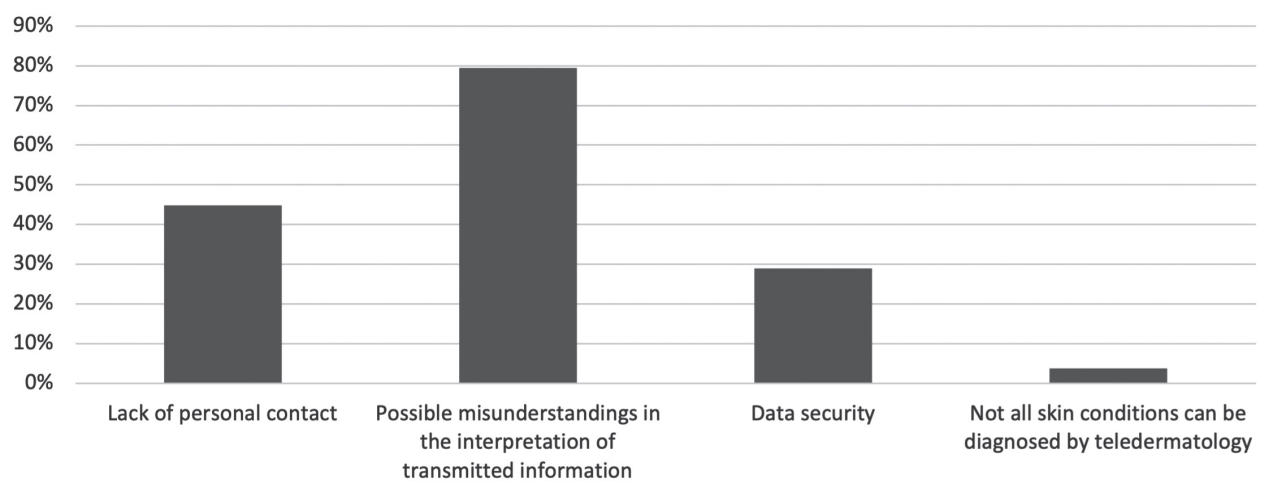

Figure 3 - Disadvantages of teledermatology.

to implementing TD, especially because rural areas without access to dermatologists are more likely to have poor internet connection. ${ }^{1}$ This was the most common reason for not using TD among Saudi dermatologists in a study conducted on 102 participants. ${ }^{10}$ Thus, establishing an infrastructure for TD is crucial to ensure patient privacy, accurate diagnosis, and utmost care to patients.

Most of our respondents (63.6\%) strongly agreed or agreed that TD should be part of residents' education programs. Similarly, another study found that $79 \%$ of residents agreed or strongly agreed. Also, residents believed that TD is most suitable to cover educational competence for "practice-based learning and improvement", followed by "medical knowledge" more than "professionalism" ${ }^{22}$ Although residents are willing to incorporate TD in their residency program, it is currently poorly incorporated. Wanat et al, ${ }^{23}$ found that only 34 programs out of 57 surveyed in the United States of America (USA) incorporated TD in residency programs, while 39 were interested in incorporating it. Practicing TD in residency will help dermatologists become more comfortable with TD in future practice. ${ }^{4}$

Study limitations. Our survey-based study revealed many aspects of the utilization of TD in KSA during the COVID-19 pandemic particularly that it compared the implementation of TD in both inpatient and outpatient setting. However, it has limitations. First, it is a survey based study that could be affected by participants' bias, which was considered as we interpreted the data. Also, the low response rate ( $\mathrm{n}=111$ responses) from 664 emails sent to dermatologists. Teledermatology implementation also differs widely between hospitals, which can affect dermatologists' perceptions. Finally, around half of the respondents are in the central region, which have relatively better facility and network connection than other areas in KSA. Thus, it can affect the dermatologists' perception regarding TD.

In conclusion, the most common way to utilize TD in our study was through phone calls, and the most important use is for a triage consult before seeing the patient. When we compared the use of TD in the outpatient and inpatient settings, outpatient responses always had more positive attitudes than inpatients. The most important advantage was to reduce the risk of COVID-19 infection. More than half of respondents use TD for diagnosis and management, and a higher number consider using it in the future. Thus, we recommend further larger-scale studies regarding patient and dermatologist satisfaction with the utilization of TD. Also, it is necessary to establish an infrastructure for TD that protects patient's privacy and ensures accurate diagnosis.

Acknowledgment. The authors gratefully acknowledge the members of Saudi Commission for Health Sciences for sending the survey email to all practicing Saudi dermatologists. Also, we would like to thank Scribendi members for english language editing.

\section{References}

1. Pearlman RL, Le PB, Brodell RT, Nahar VK. Evaluation of patient attitudes towards the technical experience of synchronous teledermatology in the era of COVID-19. Arch Dermatol Res 2021: 1-4.

2. Whited JD. Teledermatology. Med Clin North Am 2015; 99: 1365-1379.

3. Tensen E, van der Heijden JP, Jaspers MW, Witkamp L. Two decades of teledermatology: current status and integration in national healthcare systems. Curr Dermatol Rep 2016; 5: 96-104

4. Song E, Amerson E, Twigg AR. Teledermatology in medical and continuing education. Current dermatology reports 2020; 9: 136-140. 
5. WHO. Archived: WHO Timeline - COVID-19 [Internet]. 2020 [cited 25 Jun 2021]. Available from: https://www.who. int/news/item/27-04-2020-who-timeline---covid-19

6. ADD. Dermatologists can use telemedicine during COVID-19 outbreak [Internet]. 2021 [cited 2021 Jun 25]. Available from: https:/www.aad.org/member/practice/telederm/toolkit

7. Wang RH, Barbieri JS, Nguyen HP, Stavert R, Forman HP, Bolognia JL, et al. Clinical effectiveness and cost-effectiveness of teledermatology: where are we now, and what are the barriers to adoption? J Am Acad Dermatol 2020; 83: 299-307.

8. Bull TP, Dewar AR, Malvey DM, Szalma JL. Considerations for the telehealth systems of tomorrow: an analysis of student perceptions of telehealth technologies. JMIR Med Educ 2016; 2: e11.

9. Trettel A, Eissing L, Augustin M. Telemedicine in dermatology: findings and experiences worldwide - a systematic literature review. J Eur Acad Dermatol Venereol 2018; 32: 215-224.

10. Alakeel A. Acceptance of teledermatological practices: a crosssectional study of practicing Saudi dermatologists. Cureus 2021; 13: e13710.

11. Weig EA, Tull R, Chung J, Wanat KA. Inpatient teledermatology: current state and practice gaps. J Am Acad Dermatol 2020; 83: 797-802.

12. Barbieri JS, Nelson CA, James WD, Margolis DJ, LittmanQuinn R, Kovarik CL, et al. The reliability of teledermatology to triage inpatient dermatology consultations. JAMA Dermatol 2014; 150: 419-424.

13. Snoswell C, Finnane A, Janda M, Soyer HP, Whitty JA. Costeffectiveness of store-and-forward teledermatology: a systematic review. JAMA Dermatol 2016; 152: 702-708.

14. Yeroushalmi S, Millan SH, Nelson K, Sparks A, Friedman AJ. Patient perceptions and satisfaction with teledermatology during the COVID-19 pandemic: a survey-based study. J Drugs Dermatol 2021; 20: 178-183.
15. Asabor EN, Bunick CG, Cohen JM, Perkins SH. Patient and physician perspectives on teledermatology at an academic dermatology department amid the COVID-19 pandemic. J Am Acad Dermatol 2021; 84: 158-161.

16. Statistical Yearbook - Ministry of Health [Internet]. 2020 [cited 20 Nov 2020]. Available from: https://www.moh.gov.sa/en/ Ministry/Statistics/book/Pages/default.aspx.

17. Elsaie ML, Shehata HA, Hanafi NS, Ibrahim SM, Ibrahim HS, Abdelmaksoud A. Egyptian dermatologists attitude toward telemedicine amidst the COVID19 pandemic: a cross-sectional study. J Dermatolog Treat 2020: 1-7.

18. Eber EL, Janda M, Arzberger E, Hofmann-Wellenhof R. Survey on the status of teledermatology in Austria. J Dtsch Dermatol Ges 2019; 17: 25-31.

19. Campagna M, Naka F, Lu J. Teledermatology: an updated overview of clinical applications and reimbursement policies. Int J Womens Dermatol 2017; 3: 176-179.

20. Gabel CK, Nguyen E, Karmouta R, Liu KJ, Zhou G, Alloo A, et al. Use of teledermatology by dermatology hospitalists is effective in the diagnosis and management of inpatient disease. J Am Acad Dermatol 2021; 84: 1547-1553.

21. Kaliyadan F, Amin TT, Kuruvilla J, Ali WH. Mobile teledermatology--patient satisfaction, diagnostic and management concordance, and factors affecting patient refusal to participate in Saudi Arabia. J Telemed Telecare 2013; 19 : 315-319.

22. Boyers LN, Schultz A, Baceviciene R, Blaney S, Marvi N, Dellavalle RP, et al. Teledermatology as an educational tool for teaching dermatology to residents and medical students. Telemed J E Health 2015; 21: 312-314.

23. Wanat KA, Newman S, Finney KM, Kovarik CL, Lee I. Teledermatology education: current use of teledermatology in US residency programs. J Grad Med Educ 2016; 8: 286-287. 